\title{
Introduction to Special Issue on Music and Shape
}

\author{
DANIEL LEECH-WILKINSON \\ King's College London \\ MATS B. KÜSSNER \\ King's College London
}

THIS special issue on relationships between music and shape consists of nine target articles and seventeen commentaries which, via a call for papers, have been developed in response to a conference held in London in July 2012 on 'Music \& Shape'. The conference, in turn, was the final public event of a three-year research project on 'Shaping Music in Performance', directed by Daniel Leech-Wilkinson at King's College London and part of the Centre for Musical Performance as Creative Practice (CMPCP), led by John Rink and funded by the UK's Arts and Humanities Research Council. Further details may be found at www.cmpcp.ac.uk.

'Shaping Music in Performance' grew out of the observation that performers habitually use the concept of shape when talking about the process of generating expressive performances. One of the project's early studies, by Helen Prior, showed that $90 \%$ of participants (231 musicians at various levels of expertise from 31 countries) used the concept of shape when thinking about how to perform music and $80 \%$ when talking to others about how to perform. A majority used shape in relation to notes, rhythms, melodic lines and patterns, harmonic patterns, loudness, tempo, phrases, movements and whole pieces, bodily gestures in relation to performance, expressivity, meaning, narrative, tension, mood, feeling and emotion, and images conjured up by music. Clearly a word that fulfilled so many roles for musicians, despite being drawn from a different domain than sound, had something to teach us about musical response. The fact that on the face of it sound and shape, in everyday life, appear to be unrelated only made it more intriguing.

The 'Shaping Music in Performance' team - Daniel Leech-Wilkinson, Helen Prior, Dan Tidhar and Mats Küssner - took several approaches, including questionnaires, interviews, drawing tasks, and gesture capture. But we also held a number of workshops for speakers from beyond the project itself, culminating in the three-day conference in London. The idea was to gather as many different views of the music-shape relationship as we could, hoping to add as much as possible to the understanding of the nature of the relationship which was beginning to emerge from the project.

The nine target articles are grouped - rather loosely - into three broader themes. In Pedagogy and Performance the reader is invited to explore the relationship between the shape of gestures and sonic events in vocal lessons of South Indian Karnatak music; the use of musical shaping gestures in rehearsal talk by performers with different levels of hearing impairment; and what it means for professional DJs to shape a set on their turntables.

In Motion Shapes the reader is invited to discover how motiongrams can be used to sonify the shape of human body motion; how pianists' shapes of motion patterns embody musical structure; and how mathematical techniques can be used to quantify shapes of real-time visualizations of sound and music.

In Perception and Theory the reader is invited to learn about cross-cultural representations of musical shapes from the UK, Japan and Papua New Guinea; the evolutionary origins of tonality as a system for the dynamic shaping of affect; and how shaping and coshaping of 'forms of vitality' in music gives rise to aesthetic experience.

The variety of views represented in this special issue of EMR only emphasises the remarkable flexibility and reach of shape as a way of thinking about how music is experienced and practised over time. Indeed, its usefulness to researchers as well as to musicians strongly suggests that it may be a key to enhancing understanding of important aspects of music perception and response. The issue will be most successful if it leads on to further work elucidating the ease with which shape allows us to speak of music's effects.

We are very grateful to EMR's editors Nicola Dibben and Renee Timmers for giving us the opportunity to realize this special issue on music and shape, and to all authors of target articles and commentaries for their thought-provoking contributions which made working on its final form an enjoyable endeavour. 
